Daimon. Revista Internacional de Filosofía, Suplemento 5 (2016), 489-495

ISSN: 1130-0507 (papel) y 1989-4651 (electrónico)

http://dx.doi.org/10.6018/daimon/270011

\title{
Transhumanismo: hacia un nuevo cuerpo
}

\author{
Transhumanism: Towards a new body
}

JONATHAN PIEDRA ALEGRÍA*

\begin{abstract}
Resumen: Inicialmente, se analiza la concepción del cuerpo a partir de la teoría transhumanista, con la intención de mostrar como el cuerpo es el inicio y al mismo tiempo, el centro de la mejora tecnológica humana. Indicando como el potenciamiento del mismo, es un paso fundamental para la superación de las limitaciones biológicas humanas. El trabajo finaliza planteando algunas críticas a la epistemología que subyace a la concepción del cuerpo transhumanista.

Palabras claves: transhumanismo, tecnología, mejoras corporales, mejora, potenciación.
\end{abstract}

\begin{abstract}
Initially, the conception of the body is analyzed from the transhumanist theory, intended to show how the body is the beginning and at the same time, the center of human technological improvement. Indicating as the enhancement thereof, it is fundamental to overcoming human biological limitations step. The paper concludes by raising some criticisms of the epistemology underlying the concept of transhumanist body.

Keywords: transhumanism, technology, body modifications, improvement, enhancement.
\end{abstract}

\section{Transhumanismo (Humanity+)}

El transhumanismo (de ahora en adelante $\mathrm{H}+$ ) es una postura de carácter científico y filosófico que considera que la condición humana puede ser potenciada y mejorada por los avances tecnológicos. De manera amplia, el movimiento transhumanista se considera como deudor del humanismo secular e ilustrado, ya que el $\mathrm{H}+$ confía decididamente en que el poder de la razón y el progreso material son los motores que conducirán a la humanidad hacia un perfeccionamiento biológico, a través de los avances de la ciencia y la tecnología. Todo esto con la intención de lograr la plenitud y la felicidad de los seres humanos. Según el H+, en el período histórico que vivimos "es obligatorio tener en cuenta la posibilidad de un progreso tecnológico dramático. Sería trágico si no se materializaran los potenciales beneficios a causa

Fecha de recepción: 08/06/2016. Fecha de aceptación: 25/07/2016.

* Universidad Nacional de Costa Rica. Escuela de Filosofía. Académico e Investigador. Correo electrónico: jonathan.piedra.alegria@una.cr. Áreas de investigación: Filosofía de la Tecnología, posmodernidad, fillosofía de la psicología y ciencias cognitivas, transhumanismo, posthumanismo, pensamiento nietzcheano, human enhancement. Algunas publicaciones recientes: Nihilismo capitalista (Revista Praxis. Número 68-69. Universidad Nacional de Costa Rica) Re-presentando a la vida: Artaud y la posmodernidad. (Revista Praxis. Número 71. Universidad Nacional de Costa Rica.) 
de una tecnofobia injustificada y prohibiciones innecesarias." (Declaración Transhumanista). A partir de consideraciones como las anteriores, se valora al actual ser humano (homo sapiens) no como el estado más elevado y consumado de la filogénesis de nuestra especie, y por lo tanto tampoco lo más valioso (lo cual implicaría un especismo injustificado, según Peter Singer) sino como un estado que puede y debe ser superado. Para esto, se deben mejorar y potenciar ciertos aspectos que se relacionan con la calidad de vida, extender la esperanza de esta, disminuir el dolor, erradicar los padecimientos o, mejorar la funcionabilidad del cuerpo, así como potenciar nuestras capacidades cognitivas (tales como la memoria o la inteligencia). El H+ implica una amplia gama de posiciones que oscilan desde lo más utópico (algunas bastante estrafalarias y en ciertas ocasiones, francamente disparatas) e ingenuamente optimistas (optimismo dinámico $^{1}$ ) en donde la tecnología desaparecerá la vejez o muerte. Algunos transhumanistas consideran que algunas de estas modificaciones terminarán en una conversión moral de humanidad en donde la mayor parte de la población humana será virtuosa. También encontramos otras posturas más realistas y concretas que consideran que los problemas sociales y existenciales nunca desaparecerán, aunque la tecnología y la ciencia nos ayudarán bastante a sobrellevarlos.

El fundador del Instituto Extropiano, Max More (2005) nos muestra claramente esto cuando indica que:

El "transhumanismo" es un término general dado a la escuela de pensamiento que se niega a aceptar las limitaciones humanas tradicionales como la muerte, la enfermedad y otras debilidades biológicas. Los transhumanistas están típicamente interesados en una variedad de temas futuristas, incluida la migración espacio, la transferencia mental y la suspensión criogénica. Los transhumanistas también están muy interesados en temas más inmediatos, como la biotecnología y la nanotecnología, la informática y la neurología. ${ }^{2}$ (La traducción es mía)

Lo cierto del caso es que la mayoría de quienes promueven el $\mathrm{H}+$ (como el Jefe de ingenieros de Google) comparten de alguna forma este futurismo, al tiempo que promueven, la mayoría de las veces, posiciones liberales. Según Vaccari (2013) el transhumanismo es un movimiento que se origina a partir de los presupuestos económicos sociales del mundo anglosajón, por lo que muchos de sus tópicos centrales, reflejan, en algún sentido los ideales de libre mercado, así como una ética utilitarista tan defendida en países como los Estados Unidos. Ya sea este el caso o no, lo que no se puede negar es que el ideal de progreso científico y mejora tecnológica-humana, subyacen en la mayorías de la disciplinas y posturas desarrollistas actuales. No en vano, cada vez con más frecuencia los países desarrollados, así como los que no lo son, invierten en la investigación tecno-científica, específicamente en lo relacionado con la innovación y el diseño (I+D), como formas de crear progreso económico y en algunos países, avance social.

1 Un optimismo dinámico según More en More \& Vita-More (2013).

2 "Transhumanism" is a blanket term given to the school of thought that refuses to accept traditional human limitations such as death, disease and other biological frailties. Transhumans are typically interested in a variety of futurist topics, including space migration, mind uploading and cryonic suspension. Transhumans are also extremely interested in more immediate subjects such as bio- and nano-technology, computers and neurology." More, M. http://www.mactonnies.com/trans.html 
El artículo tercero de la Declaración Transhumanista señala que "Los transhumanistas creemos que siendo generalmente receptivos y aceptando las nuevas tecnologías, tendremos una mayor probabilidad de utilizarlas para nuestro provecho que si intentamos condenarlas o prohibirlas.". De esto se puede desprender que el elemento más importante para cualquier enfoque transhumanista es el desarrollo y la aplicación de la tecnología. En este sentido, sigue la línea ya mencionada del humanismo ilustrado, al considerar a la ciencia (tecnociencia en este caso) como un aliado fundamental en su proyecto civilizatorio.

Siendo esto así, en el H+ subyacería un humanismo tecnológico que no dista cualitativamente de los humanismos tradicionales que buscan transformar al ser humano a partir de un refinamiento cultural, una educación determinada o la imposición de ciertos valores éticos. No obstante, sería importante matizar esta afirmación ya que el transhumanismo posee un elemento bastante distintivo. El H+ se diferencia de todos ellos en tanto no busca utilizar la tecnología desde un punto de vista social o político, para mejorar las condiciones materiales (y por lo tanto alargar la vida, por ejemplo) o para distribuir mejor la riqueza de los países (lo que implicaría una mejor calidad de vida). Tampoco plantea utilizar los aportes de la ciencia y la tecnología para reducir el efecto invernadero u optimizar los servicios de salubridad (lo cual sin duda traería una reducción de los padecimientos o dolores). Asimismo, tampoco se interesan en la utilización de las tecnologías de la comunicación e información (TICs) para mejorar la educación, sino que la mejora tecnológica humana se plantea en un plano estrictamente individual (independientemente de que algunos autores transhumanistas mencionen algunos de estos puntos de manera accesoria o indirecta en sus textos). La idea es que este potenciamiento personal, termine a la postre, en la mejora de la humanidad como un todo. "El transhumanismo (...) no sólo promueve los medios tradicionales de mejora de la naturaleza humana (...) sino también la aplicación directa de la medicina y la tecnología para superar algunos de nuestros límites biológicos básicos." (Bostrom, 2005b, pp. 4)33.

Es así, como el mejoramiento y la potenciación humana (human enhancement) se formulan desde una base rigurosamente individual, a diferencia del resto de los humanismos clásicos que tenían como punto de partida un contexto social más amplio desde donde se debía orientar al ser humano.

\subsection{Mejoramiento Tecnológico Humano}

Toda esta situación nos plantea un primer problema: ¿Qué quiere decir mejorar para el H+? Según Azevedo (2013):

las mejoras son las intervenciones en los subsistemas orgánicos que no tienen por objeto prevenir, promover o rehabilitar a las personas cierta capacidad de evitar enfermedades graves, trastornos o discapacidades (...) que tienen como objetivo mejorar las capacidades más allá de las necesidades humanas básicas o de forma absoluta básica el bienestar humano, incluyendo la salud (pp. 70).

3 "Transhumanism (...) it promotes not only traditional means of improving human nature, such as education and cultural refinement, but also direct application of medicine and technology to overcome some of our basic biological limits." La traducción es mía. 
Esto quiere decir que el mejoramiento de los seres humanos no se deriva de algún concepto tradicional de salud, de manera que, en principio, parte de un ámbito no médico. Por lo tanto no implica directamente, tratamientos ni procedimientos correctivos para problemas de salud o padecimientos propios del envejecimiento. Aunque estos de alguna forma se encuentran implicados dentro del pensamiento transhumanista, como parte de sus ideales.

Existe una gran ambigüedad en la literatura en cuanto al human enhancement ya que en el fondo la diferenciación entre lo que es una mejora y lo que no lo es, se vuelve bastante borrosa y en el fondo arbitraria. Sirva de ejemplo para esto, la distinción que realiza Ida (en Bostrom \& Savelecsu 2008) entre las mejoras naturales (natural improvement), a la manera en cómo se pueden mejorar nuestras físicas por el entrenamiento riguroso, y las mejoras innaturales (unnatural improvement) que tienen como finalidad superar los límites biológicos naturales de la especie humana como resultado de las intervenciones tecnológicos.

Nicholas Agar (2004) plantea otra taxonomía cuando se refiere a la distinción entre las mejoras radicales, las cuales, según él, tendrían consecuencias desastrosas para la humanidad, ya que implicarían cambios profundos en lo que es ser humano (i.e, la clonación o manipulación del genoma humano) y las mejoras moderadas que no implican cambios constitutivos en el ser del humano, como, por ejemplo, todas aquellas que implican cambios anatómicos no profundos.

No obstante, una característica que comparten la mayoría de las distinciones al respecto, es que una mejora tiene la característica de superar algún tipo de límite (biológicos en este caso), lo que convierte al human enhancement en algo potencialmente ilimitado, constreñido solamente por los materiales o por el desarrollo tecnológico actual. Esto es relevante ya que el human enhancement al estar basado epistemológicamente por argumentos diferentes a la curación o terapia, no involucra un estado final o de perfección, ya que la mejora está pensada en función de la superación de los límites biológicos de la especie y de ahí pasar a un estado posthumano (H++).

$\mathrm{El} \mathrm{H+,} \mathrm{promueve} \mathrm{principalmente} \mathrm{las} \mathrm{modificaciones} \mathrm{que} \mathrm{impliquen} \mathrm{beneficios} \mathrm{intrínsecos}$ o externalidades positivas netas (Bostrom, 2003) tales como abolir el sufrimiento propio de las enfermedades (por medio de neuro-tecnología, por ejemplo), ralentizar el proceso de la vejez o el prolongamiento de la vida. Estas mejoras son vistas como algo bueno en sí, sin importar el contexto, ya que no son algo positivo basado en la comparación de un ideal o un modelo (i.e ser más alto o más delgado) sino que su beneficio radica en la potenciación de características que no ocupan una valoración subjetiva. ¿A quién no le gustaría no enfermarse nunca? O ¿no sentir dolores físicos? Pues bien, este tipo de mejoras son las que busca el $\mathrm{H}+$.

\subsection{Superando el límite: El cuerpo 2.0}

Inicialmente, el lugar por excelencia, donde se debe aplicar el enhancement es el cuerpo. En él encontramos: el dolor, el cansancio, la enfermedad, la vejez y en definitiva la muerte. En la posición que estamos comentando, el cuerpo adopta las clásicas consideraciones cartesianas que lo suponen únicamente desde su aspecto de res extensa. El cuerpo es un (bio) material que interactúa a nivel físico y biológico dentro de una determinada forma-anatomía siguiendo principios funcionales. Esto es en principio, lo que permite que el cuerpo pueda ser 
cambiado, potenciado y mejorado. Ya que, si lo principal del cuerpo es que se puede medir a partir de sus de sus funciones, esto permite ponerlo al mismo nivel ontológico del resto de los seres no-humanos, permitiendo la interacción con máquinas, modificarlo genéticamente, relacionarse con sistemas de información o potenciarlo con nanotecnología, por mencionar solo algunos ejemplos. Esto abre una cantidad infinita (teóricamente) de posibilidades en las cuales el cuerpo, puede ser mejorado por la tecnología.

Natasha Vita-More, una famosa transhumanista, comenta esto con bastante entusiasmo cuando indica que:

El transhumano es una etapa evolutiva del ser exclusivamente biológico para convertirse en un ser post-biológico. Post-biológico significa un derramamiento continuo de nuestra biología y la fusión con las máquinas. (...) El cuerpo, a medida que nos transformamos a nosotros mismos a través del tiempo, se enfrentará a diferentes tipos de apariencias y diseños y materiales. (...) Para el senderismo de montaña, me gustaría que me extendieran la fuerza de las piernas, la resistencia, una piel-envoltura para protegerme de los aspectos ambientales dañinos, auto-hidratación, capacidad enfriamiento, audición extendida y visión aumentada (...). Para una fiesta, me gustaría una mirada ecléctica - una piel de bronce brillante con reflejos de color verde esmeralda, un aumento de la altura por encima de otras personas, un sofisticado sistema de sonido interno de modo que pudiera alterar la música para satisfacer a mi propio gusto, un dispositivo de mejora de la memoria, un selección emocional para sentirme bien con la gente, así que no llegaría arrastrada a conversaciones inapropiadas-. Y un oído parabólico para poder escuchar las conversaciones a través del cuarto, si en la que yo estoy empieza a decaer ${ }^{4}$. (La traducción es mía)

El cuerpo se convierte sin duda en el lugar para la mejora, siempre y cuando la funcionabilidad corpórea no se vea disminuida, ya que esto evidentemente implicaría un detrimento, es decir, lo opuesto del enhancement. El cuerpo se torna, de esta manera en la materia prima en donde operara la mejora, al suprimirle, las limitaciones físico-biológicas.

Esto platea que la esfera somática (otra manera en como los transhumanistas llaman al cuerpo) sea el campo simbólico-material que muestre el dominio del ser humano, sobre su precariedad y su condición misma de humano. Ya que, por medio de la ciencia y la tecnolo-

4 "A transhuman is an evolutionary stage from being exclusively biological to becoming post-biological. Post biological means a continuous shedding of our biology and merging with machines. (...) The body, as we transform ourselves over time, will take on different types of appearances and designs and materials. (...) For hiking a mountain, I'd like extended leg strength, stamina, a skin-sheath to protect me from damaging environmental aspects, self-moisturizing, cool-down cap ability, extended hearing and augmented vision (...). For a party, I'd like an eclectic look - a glistening bronze skin with emerald green highlights, enhanced height to tower above other people, a sophisticated internal sound system so that I could alter the music to suit my own taste, memory enhance device, emotional-select for feel-good people so I wouldn't get dragged into anyone's inapropriate conversations. And parabolic hearing so that I could listen in on conversations across the room if the one I was currently in started winding down". Vita More N. Who are transhumans? http://www.transhumanist.biz/ interviews. htm. Es interesante notar que ninguna de estas modificaciones podrían ser consideradas dentro de la categoría de beneficios intrínsecos propuestos por Bostrom y que según él, son los que promueve el transhumanismo. No obstante, estas divergencias vienen a mostrar, lo que indicamos anteriormente respecto a la disparidad de opiniones con respecto a lo que es mejorar. 
gía los seres humanos podemos superar nuestra situación e inclusive dirigir voluntariamente la evolución de nuestra especie. Esta Evolución Dirigida (Directed Evolution) es uno los principales (y más imaginativos) postulados del transhumanismo, ya si nuestra historia filogenética y por la tanto la evolución del ser humano hasta el punto actual, han sido un proceso sumamente extenso, la tecnología en este momento (según muchos transhumanistas) nos abre posibilidades (impensables en otros momentos históricos), que nos permitirían ser verdaderamente maestros de nuestro propio destino, superando este fatum biológico.

\section{Limitando la superación: Discusión y crítica}

A partir de las posiciones transhumanistas sobre el cuerpo, es claro que algunas de las principales críticas que puede afrontar provienen, vienen no solo desde el ámbito ético o social, sino desde los enfoques epistemológicos que subyacen en buena parte de estas posturas. En primer lugar (1), es claro que una de las ideas que sustentan al H+ como lo es la mejora tecnológica humana, se encuentra respaldada en una instrumentalización del cuerpo humano bastante cosificadora, producto de un biologicismo-reduccionista. El cuerpo sigue la explicación mecánica de la naturaleza humana que se ha venido dando de manera paulatina (aunque progresiva) en los últimos dos mil años. Lo cual no parece ser para nada un avance con respecto a las posturas más tradicionales respecto al ser humano. Esto implica que la comprensión sobre el ser humano del $\mathrm{H}+$, contrariamente, a lo que se podría pensar, parte de las viejas concepciones ontológicas y metafísicas que han gobernado a Occidente por mucho tiempo. Es decir, el transhumanismo, sigue siendo enormemente conservador, muy a su a pesar.

Esto se muestra patentemente cuando se arroga la mejora tecnológica humana desde un determinismo no cuestionado en el fondo, sino aceptado de forma a priori. Esto se debe, eventualmente, a que una parte importante de la literatura sobre el H+ dedica su argumentación únicamente en los posibles resultados de la aplicación de la mejora tecnológica del ser humano, pero toman poco tiempo en cuestionar sus propias sus concepciones epistemológicas y metodológicas. Esto también se pasa muchas veces por alto, ya que quienes se oponen al H+ ("bioconservadores" como algunos les llaman) se dedican exclusivamente a aspectos éticos (o bioéticos) puesto que los consideran primordiales en este debate, posiblemente con razón. Aunque no son los únicos.

Ciertamente esta concepción reduccionista no solo se presenta en el H+, sino también en teóricos de la inteligencia artificial $^{5}$, en neurocientíficos y en general en lo que respecta a la divulgación científica actual, por lo que no sería correcto adecuar esto como un fenómeno que se da de manera especial o con mayor presencia en el $\mathrm{H}+$.

Sin embargo, también es cierto, que esta situación impide ver que en enfoques como estos (sobre todo en lo concerniente a esta epistemología naturalizada) se olvida la integralidad del cuerpo, así como la estrecha relación de coexistencia y con-formación entre la mente y la corporalidad. Inclusive, se dejan de lado los descubrimientos más recientes sobre cognición encarnada o enactivismo que muestran que el cuerpo no es solo un hardware muy complejo o simplemente un material más en su sentido únicamente objetual. Esto quizá no

5 Cf. Por ejemplo las críticas de Hubert Dreyfus en su libro What Computers Still Can 't Do. A Critique of Artificial Reason. 
sea un problema a lo interno de lo teoría transhumanista desde un punto de vista amplio (nuestra posición es que sí lo debería ser, como lo hemos venido indicando), ya que es consecuente con sus propuestas, favoreciendo de una manera falaciosa las intervenciones y comentarios de los supuestos expertos en el tema (i.e ingenieros, expertos en computación y más recientemente cualquier rama disciplinar que tenga el prefijo neuro en su nombre), que casi siempre desechan muchos de estos cuestionamientos. En este sentido, el transhumanismo tampoco parece ser muy novedoso. Nuevas odres para el mismo vino.

Igualmente, es importante mostrar que, esto en sí mismo, no es una objeción a la idea de mejora tecnológica humana como tal, aunque si plantea aspectos debatibles para ella en el contexto transhumanista, que serían importantes desarrollar sobre el estatuto epistemológico que sirve como base y fundamento al transhumanismo. Puesto que el $\mathrm{H}+$ toma el human enhancement en sentido amplio, y lo reduce a un medio pasivo en el cual se circunscribe la capacidad modificadora del agente racional por medio de la tecnología, lo cual al menos desde nuestra posición limita la capacidad simbólica-transformativa del enhacement, ya que la traduce únicamente a una relación instrumental y no verdaderamente transformadora, ya que la mejora siempre trabajaría sobre la vieja ontología clásica, sin crear ningún verdadero cambio, al menos en esta.

\section{Referencias bibliográficas}

Agar, Nicholas. (2004). Liberal Eugenics: in defence of human enhancement, Oxford, Blackwell Publishing.

Agar, A. (2010) Humanity's End: Why We should Reject Radical Enhancement, Cambridge: MA, MIT Press.

Azevedo, Marco. (2013). Human Enhancement: A new issue in philosophical agenda. Principios Revista de Filosofía. Natal (RN) v.20, n.33. Janeiro/Junho de 2013. Pp. 265-303

Bostrom, Nick. (2003). "Human Genetic Enhancements: A Transhumanist Perspective." Journal of Value Inquiry.

Bostrom, Nick. (2005). "A history of Transhumanist Though" Journal of Evolution and Technology. $14 \mathrm{~N}^{\circ} 1$ (april).

Bostrom, Nick. (2005b) Transhumanist values. Review of Contemporary Philosophy, Vol. 4, May.

More, Max \& VITA-MORE, Nathasa. (2013) The transhumanist reader. John Wiley \& Sons, Inc. USA.

More, Max. http://www.mactonnies.com/trans.html Consultado el 2 de febrero del 2014

Savelescu, Julian. \& BOSTROM, Nick. (2007) Human Enhancement, Oxford, Oxford University Press, The Transhumanist Declaration. http://humanityplus.org/philosophy/ transhumanist-declaration/ Consultado el 2 de febrero del 2015.

Vaccari, Andrés. (2013). La idea más peligrosa del mundo: Hacia una crítica de la antropología transhumanista. Tecnología y Sociedad. 1 (2). pp. 39-59.

Vita-More, Nathasha. (2000) Who are transhumans? http://www.transhumanist.biz/ interviews.htm Consultado el 2 de febrero del 2014.

Velázquez, Héctor. (2009) Transhumanismo, Libertad e Identidad Humana Thémata. Revista de Filosofía. Número 41. Pp. 577-590. 
